\title{
EXPERIMENTAL DAMAGE ASSESSMENT OF SUPPORT CONDITION FOR PLATE STRUCTURES USING WAVELET TRANSFORM
}

\author{
Muyideen AbdulKareem \\ Construction Research Centre, Universiti Teknologi Malaysia, Johor, Malaysia \\ School of Civil Engineering, Universiti Teknologi Malaysia, Johor, Malaysia \\ e-mail: moabdulkareem2@live.utm.my \\ NORHISHAM BAKHARY \\ School of Civil Engineering, Universiti Teknologi Malaysia, Johor, Malaysia \\ Institute of Noise and Vibration, Universiti Teknologi Malaysia, Kuala Lumpur, Malaysia \\ MOHAMmadreza VAFAeI \\ School of Civil Engineering, Universiti Teknologi Malaysia, Johor, Malaysia \\ Forensic Engineering Center, Universiti Teknologi Malaysia, Johor, Malaysia \\ Norhazilan Md Noor, Roslida Abd Samat \\ School of Civil Engineering, Universiti Teknologi Malaysia, Johor, Malaysia
}

\begin{abstract}
Wavelet transforms (WTs) have gained popularity due to their ability to identify singularities by decomposing mode shapes of structures. In VBDD, the support condition of a structure influences structural responses and modal properties. In fact, the structural responses and modal properties are a lot more sensitive to changing boundary conditions than to crack and fatigue damage, resulting in inaccurate damage detection results. Therefore, in this study, sensitivity tests to estimate a suitable distance range which allows damage detection by imposing single support damage are carried out. The estimated appropriate distance is then applied to detect damage at multiple supports. This involved the applicability of response acceleration of plate structures to support assessment by applying continuous wavelet transform (CWT) and discrete wavelet transform (DWT). The damage cases have been introduced by releasing bolts at specified fixed supports of the plate to simulate the damage. The response accelerations of the rectangular plate at points close to the supports were measured and decomposed using CWT and DWT to assess the structural integrity of each support. The results showed that an appropriate distance range was necessary for accurate damage detection, and both, CWT and DWT could provide reliable outputs. However, the first- and fourth-level detail coefficients of DWT failed to indicate damage in some cases. A more detailed investigation of the effect of different wavelet scale ranges on damage detection using CWT demonstrated that the accuracy of damage detection increased as the scale decreased.
\end{abstract}

Keywords: support, damage, plate, continuous wavelet transform, discrete wavelet transform

\section{Introduction}

Structural damage is often caused by continuous exposure of structures to extreme conditions, resulting in increased maintenance costs. In simple structures, such damage is conventionally detected and located by visual inspection. However, owing to the size and complexity of modern structures, visual inspection and other non-destructive techniques (NDTs) require a considerable amount of time and access information for damage identification. These drawbacks have hastened the need for efficient vibration-based damage detection (VBDD) techniques for damage identification in structures. Such techniques aim at reducing the operational and maintenance 
cost of a structure even after it exceeds the designed lifespan. They provide information for damage identification by measuring dynamic responses (acceleration, velocity, and displacement), derived modal parameters (frequency, mode shape, modal damping), and their derivatives estimated before, during, and after damage occurrence in the structure.

Many researchers have employed wavelet transforms (WTs) as a tool for damage identification in structures using VBDD techniques (Liu et al., 2014), because WTs decompose signals into summation of time-domain basis functions of various frequency resolutions, which provide information about the signal in both time and frequency domains, and this gives an edge over the change in frequency (Bakhary et al., 2010). This facilitates extraction of information from non-stationary signals, e.g., earthquake acceleration, which can resolve changes in the frequency content as a function of time (Yu et al., 2016). Unlike the Fourier Transform (FT), which projects a signal only in the frequency domain and is, thus, limited to stationary signals, WTs have the ability to detect singularities in signals that are not visible to the human eye. This property has been exploited by researchers to detect damage in structures.

The efficiency of WTs in identifying damage in civil structures has been demonstrated by several researchers. This includes analysis of structural responses (Zhong and Oyadiji, 2011), lamb waves (Chen et al., 2017) and acoustic emission (Zhang et al., 2015). WT analysis of mode shapes and structural responses of damaged structures indicates perturbations in the decomposed signal, and these perturbations indicate damage characteristics. For example, Lee et al. (2000) demonstrated the ability of WTs to identify damage by decomposing fundamental mode shapes of a beam. They employed only the mode shapes of numerical and experimental models of the damaged beam and applying the concept of vanishing moments of wavelet functions. Later on, Hong et al. (2002) identified damage in a beam by mode shape wavelet transformation in terms of the Lipschitz exponent. Douka et al. (2003) applied symmetrical 4 wavelet with two vanishing moment to identify a crack in a cantilever beam. The size of the crack was estimated by relating the wavelet coefficient to an intensity factor. Furthermore, Hester and González (2012) used response acceleration to identify damage in a bridge by WT analysis of the measured acceleration time history. The average wavelet content of the acceleration was estimated for each strip of the bridge and damage was detected by comparing the energy content of the damaged and undamaged strips when the appropriate scale ranges were selected. The results demonstrated that the damaged strips had higher energy content than the undamaged strips. The WT techniques was shown to be efficient in damage identification in many other studies (Yang and Nagarajaiah, 2014; Cao et al., 2014; Cantero and Basu, 2015).

Despite the efficiency of WTs in damage identification, few studies have investigated the application of WTs to the assessment of structure supports. The support condition influences the structural responses and modal properties, in fact, the structural responses and modal properties are more sensitive to changing boundary conditions than to damage in the structure. Thus, a change in the support condition may lead to inaccurate health monitoring results when damage is being investigated. The above existing WT methodologies are incapable of detecting damage in structural supports, since mode shape decomposition for damage detection is unable to provide information on the structural integrity of the structure support.

In this study, sensitivity tests to estimate a suitable distance range which allows damage detection by imposing single support damage have been carried out. The estimated appropriate distance was then applied to detect damage at multiple supports. This involved the applicability of response acceleration of plate structures to support assessment by applying the continuous wavelet transform (CWT) and discrete wavelet transform (DWT). The applicability of this method is demonstrated through laboratory-based experimental tests on a plate structure. The sides of the plate were fixed by using bolts and nuts, and damage was induced at the boundaries by releasing bolts. The sensors were placed in three different formats by using three different distance ranges to study the sensitivity of the damage print in the measured acceleration re- 
sponses to distance between the support and sensors. This was accomplished by varying the distance between the point of sensor placement and the supports. Due to the importance of the selected wavelet scale for damage identification, the effect of wavelet scale on damage detection by using CWT was also studied.

\section{Wavelet transform}

\subsection{Discrete wavelet transform (DWT)}

WT analysis of time-varying signals is a localization analysis technique in the frequency and time domains, whereby the windows of both, time and frequency, could be altered. The discontinuity at the location of damage allows identification of structural damage. According to Staszewski (1998), a signal $x(t)$ can be represented by its approximation and detail coefficients as

$$
x(t)=A_{j}+\sum_{j \subseteq j} D_{j}
$$

where $A_{j}$ is the approximation at the level $j$

$$
A_{j}=\sum_{j \supset j} D_{j}
$$

$D_{j}$ is the detail at the level $j$

$$
D_{j}=\sum_{k \in Z} a_{j, k} \psi_{j, k}(t)
$$

$\psi_{j, k}$ is the wavelet function, $Z$ is a set of positive integers, and $a_{j, k}$ is the wavelet coefficient at the level $j$

$$
\psi_{j, k}(t)=2^{j / 2} \psi\left(2^{j} t-k\right)
$$

Equations (2.1)-(2.4) show that the signal is split into approximations $A_{j}$ and details $D_{j}$. The selection of an appropriate mother wavelet is crucial for obtaining the best damage detection results using a wavelet-based technique. This selection may be based on symmetry, regularity, and capability for precise reconstruction of the analyzed signal (Ovanesova and Suárez, 2004). Another selection criterion that can be considered is the number of vanishing moments (Zhong and Oyadiji, 2011). However, there exists a no systematic approach for selecting wavelet functions. Hence, a trial-and-error approach has been adopted to select an appropriate mother wavelet that gives the best result (Vafaei et al., 2015). After several rounds of trial and error, the Gaussian mother wavelet function was selected.

\subsection{Continuous wavelet transform (CWT)}

The CWT of a signal $f(x)$ is the integral of the product of the signal function and the complex conjugate $\psi(x)$ of the wavelet function. According to Mallat (1998), the CWT is given by

$$
W f(u, s)=\frac{1}{\sqrt{s}} \int_{-\infty}^{+\infty} f(x) \psi\left(\frac{x-u}{s}\right) d x
$$

where $s$ and $u$ are the scale and position, respectively, and $W f(u, s)$ is the wavelet coefficient of the wavelet $\psi_{u, s}(x)$. The wavelet coefficient indicates how the wavelet function correlates with the transformed signal. When there is a change in the structural stiffness, a sharp transition occurs in the dynamic response. 


\section{Methodology}

Introducing damage into a welded connection during excitation in an experimental scaled structure may not be feasible for dynamic response extraction. Hence, bolted connections were employed at the supports, similar to several studies (Li and Hao, 2016; Yu and Zhu, 2015; Yang et al., 2014). This technique is an output-only measurement technique that measures only the structural response to simulate field structures under operating conditions. Information regarding the input excitation, material, and size of the structure is not required for damage assessment. This technique relies solely on the changes imposed on the response acceleration measured at points close to the supports.

The plate was set into vibrations by using an electro-dynamic shaker, and the response acceleration of each of the three distance ratios (distance from the measurement point to the center of each support) was measured. The distances to the center of each support are given in Table 1. The experimental setup consisted of three different distance ratios: $1 / 2,1 / 4$ and l/22.4 as shown in Fig. 1a-1c, utilized one, four, and four sensors respectively. This allowed selection of the most appropriate location (amongst the three) for the sensor. The response accelerations were decomposed by using one-dimensional DWT and CWT to detect singularities in the signals. These singularities indicated the time at which the damage occurred and were used to select the exact damaged support.

Table 1. Distance ratios of the experimental setups

\begin{tabular}{|c|c|c|}
\hline Distance ratio & Actual distance $[\mathrm{mm}]$ & Number of sensors \\
\hline \hline $\mathrm{l} / 2$ & 280 & 1 \\
\hline $\mathrm{l} / 4$ & 140 & 4 \\
\hline $\mathrm{l} / 22.4$ & 25 & 4 \\
\hline
\end{tabular}

(a)

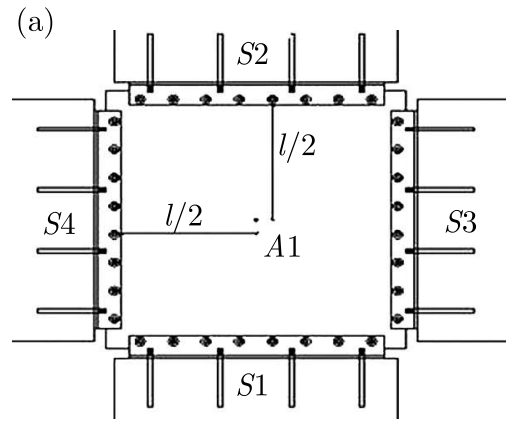

(b)

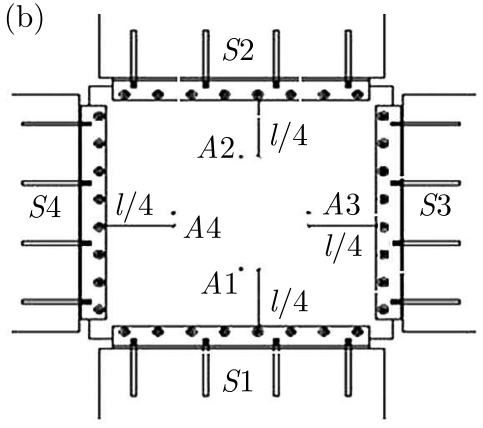

(c)

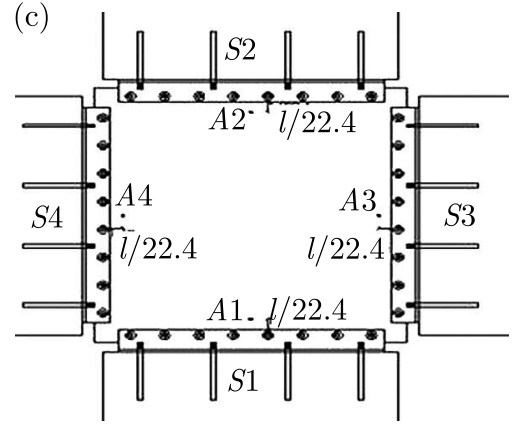

Fig. 1. Test structure showing different experimental setups: (a) $l / 2$, (b) sensor $l / 4$, (c) sensor $l / 22.4$

\section{Experimental setup and modal testing}

\section{1. $\quad$ Test structure and experimental setup}

A square steel plate was tested experimentally to demonstrate the procedure. The plate was fixed at its four sides (1, 2, 3, and 4) as shown in Fig. 1 . Its dimensions were $560 \times 560 \times 3.2 \mathrm{~mm}$. The material properties were as follows. Mass density $\rho=7836 \mathrm{~kg} / \mathrm{m}^{3}$, steel modulus of elasticity $E=195 \mathrm{GPa}$, and Poisson's ratio $\nu=0.3$. The support system of the structure was composed of eight equal steel angles (two on each side) fixed into four metal-cased concrete cuboids. Each fixed support had two steel angles facing opposite directions, forming a groove between them. The vertical legs of the equal angles were bolted into the concrete cuboids, while the horizontal legs were clamped to the sides of the plate using bolts, providing fixed supports at the plate 
four sides. The dimensions of each steel angle were $570 \times 40 \times 3 \mathrm{~mm}$, whereas each cuboid was $600 \times 300 \times 150 \mathrm{~mm}$. The weight of the concrete cuboids provided a strong boundary condition during excitation, which was required for a fixed support. The plate was fixed at each of the four sides by eight M6 bolts with a spacing of $75 \mathrm{~mm}$. These formed rigid connections at each end, representing the four fixed supports labeled as S1, S2, S3, and S4.

The experimental setup had four accelerometer sensors at maximum, namely; A1, A2, A3 and A4 with sensitivities $10.03 \mathrm{mV} / \mathrm{g}, 10.07 \mathrm{mV} / \mathrm{g}, 10.1 \mathrm{mV} / \mathrm{g}$, and $10.14 \mathrm{mV} / \mathrm{g}$, respectively, were attached to the plate. These sensors were placed on the plate at $280 \mathrm{~mm}, 140 \mathrm{~mm}$ and $25 \mathrm{~mm}$ from the center of each support. The sensors were PCB Model 352C33 (PCB Piezotronics, Inc.). The sensors monitored the acceleration of the plate at respective points under different damage scenarios. The excitation of the plate was provided by an electro-dynamic shaker (TMS; The Modal Shop, Inc.) that was mounted below the plate, and measurements were taken during excitation. A lateral channel blower (K03-MS MOR;FPZ) was connected to the electro-dynamic shaker. The data acquisition system used was Sirius Dewesoft with eight channels, running on Dewesoft software for analyzing the measured signals. The data obtained were stored in Dewesoft software and exported to Excel sheets after analysis using WT.

\subsection{Damage pattern}

Two different damage cases were considered in this study, i.e., single and multiple damage cases. The single damage case was studied first; this was done to determine the best of the three distance ratios. Following this, the best distance ratio was applied to the multiple damage case. The damage cases were defined based on the location of the damaged support. The locations considered were at support S2 for the single damage case, and supports S3 and S4 for the multiple damage case. The damage was introduced by loosening two bolts at the specified supports during excitation. The supports were considered undamaged when all the bolts were tightly fixed.

During excitation, the bolts at the support were loosened simultaneously to simulate failure that occurs during operation of the structure, which led to a change in the structural response (acceleration) of the plate at points close to the support during operation. For each damage case, the two bolts at the center of the specified support(s) were loosened simultaneously to represent a weakened (less rigid) fixed support. The removal of two bolts (out of eight) was not sufficient to cause catastrophic damage to the structure; the objective was to detect damage in its early stages to prevent destruction/damage of the structure. For the single damage case, the bolts were loosened at support S2 during the excitation. The multiple damage case involved loosening of the bolts at supports S3 and S4 at different times; the bolts at S3 were removed first, followed by the bolts at $\mathrm{S} 4$. The objective of the multiple damage case was to facilitate identification of the perturbation caused by the damage at each of the supports.

\section{Observation}

\subsection{Response acceleration}

The occurrence of damage changes the magnitude of acceleration. However, direct comparison of the measured acceleration for damage detection is rather difficult, especially when the change is obscured by noise ( $\mathrm{Li}$ and Hao, 2016). This problem was encountered in our experiment. The recorded accelerations covered the period before, during and after the bolts were removed. The recorded accelerations for the single damage case showing the sensitivity range (distance ratio) are shown in Figs. 2-4. This sensitivity range was then applied to the multiple damage case shown in Fig. 5. In Fig. 2, the response acceleration when a single sensor was installed at a distance $l / 2(280 \mathrm{~mm})$ from each support is shown. One sensor was applied there because the 
distances of the sensor from the four supports were equal. Damage was imposed on support S2 at approximately $58 \mathrm{~s}$. The recorded acceleration showed several minor peaks, but no distinctive visual perturbation could be seen in the signal. The damage print might not be visually seen in the signal, but further processing of the signal by decomposition using WT could identify such prints. In Fig. 3, the recorded response acceleration when the distance ratio was $l / 4(140 \mathrm{~mm})$ can be seen. In this case, four accelerometer sensors were installed at a distance of $140 \mathrm{~mm}$ from each support. Damage was similarly imposed at support S2 at approximately $58 \mathrm{~s}$. From the recorded acceleration, the damage at suppport S2 had an effect on the acceleration response as a change in the measured accelerations can only be seen in the signal recorded by sensor A2, as shown in Fig. 3b. This change occurred at the time of damage to suppport S2. Signals recorded by the other three sensors (A1, A3, and A4) exhibit several minor peaks. This might be due to either noise or damage. Decomposition of these signals will indicate if they actually contain the damage print or only are a result of noise.

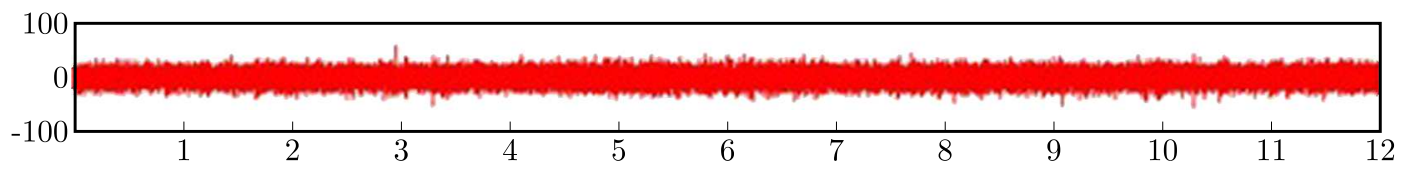

Fig. 2. Response acceleration when damage occurred at $\mathrm{S} 2$ for $l / 2$ (single damage)

(a)

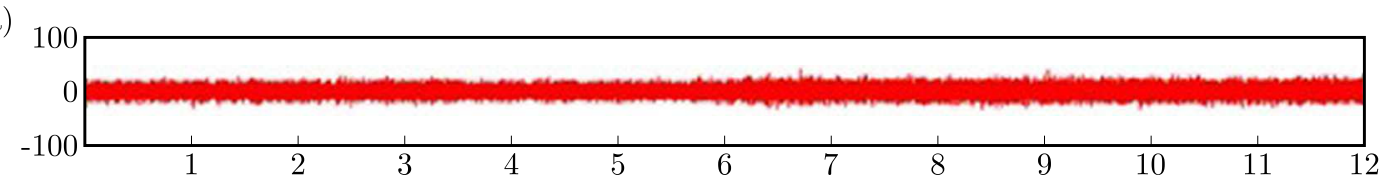

(b)

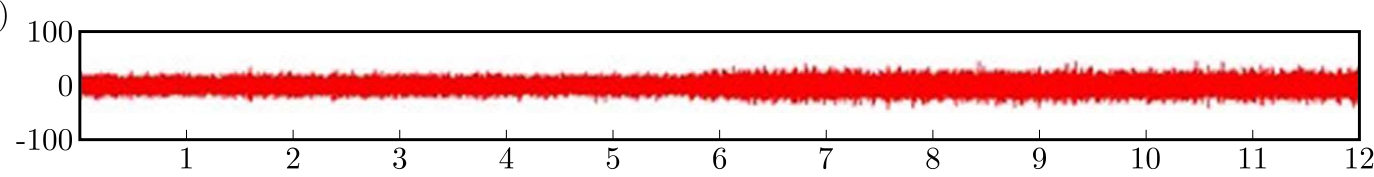

(c)

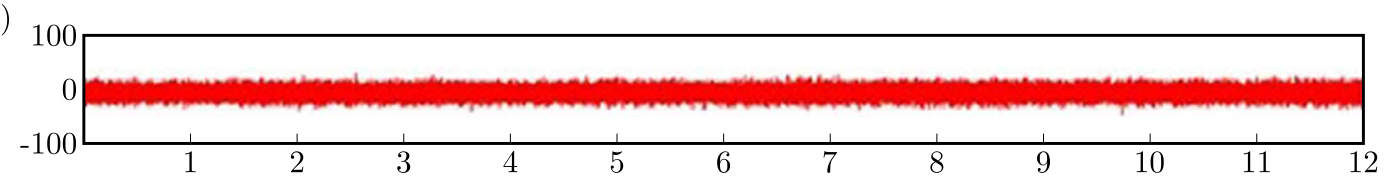

(d)

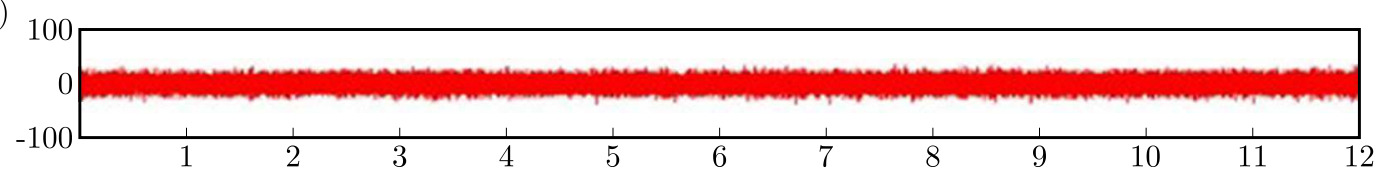

Fig. 3. Response accelerations when damage occurred at $\mathrm{S} 2$ for $l / 4$ (single damage): (a) sensor 1 ,

(b) sensor 2, (c) sensor 3, (d) sensor 4

Figure 4 shows response accelerations recorded by the four sensors at $l / 22.4(25 \mathrm{~mm})$ when damage occurred at suppport S2 (single damage case). The disturbance recorded by sensor A2 was visually observable. Damage occurred at $37 \mathrm{~s}$ and a peak was observed, indicating the occurrence of damage at the nearby support. However, several minor false peaks were also observed in the accelerations recorded by sensors A3 and A4 (Figs. 4c and 4d), indicating that noise in the measured data might lead to false damage detection. This impulse could also be caused by other sources, e.g. sudden impact by a drop weight on the plate. However, this could be differentiated from damage because a drop weight on the plate would lead to disturbance of the recorded signal by all the four sensors at the same time (impact time). Figure 5 shows the response accelerations when multiple damages occur. These damages were imposed in supports S3 and S4. The distance ratio applied there was $25 \mathrm{~mm}(l / 22.4)$. This is due to the results discussed in Section 5.2. The 
(a)

(b)
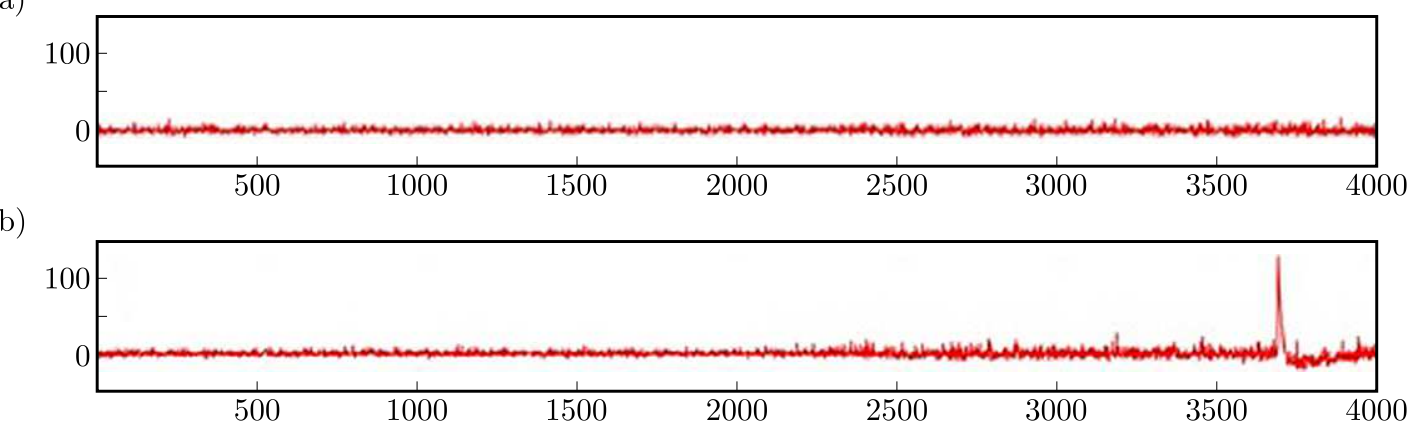

(c)

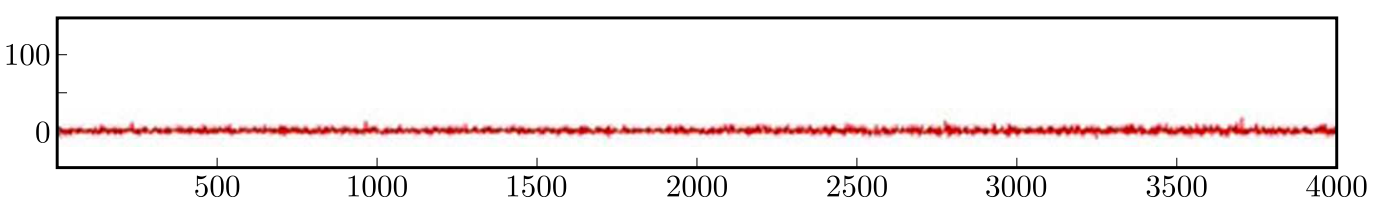

(d)

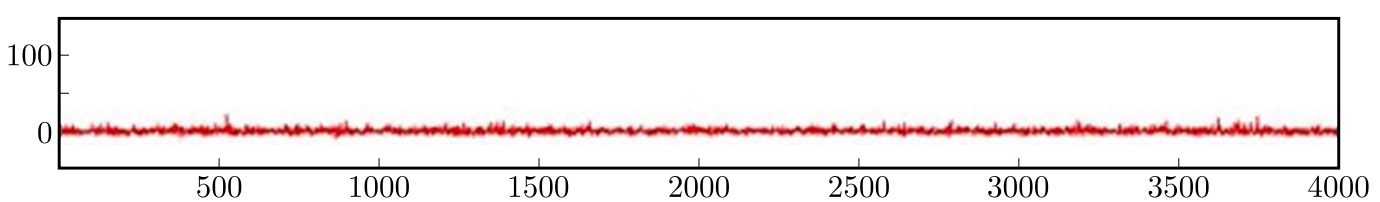

Fig. 4. Response accelerations when damage occurred at S2 for $l / 22.4$ (single damage): (a) sensor 1, (b) sensor 2, (c) sensor 3, (d) sensor 4

measurements recorded by sensors A3 and A4 (Figs. 5c and 5d) showed perturbations in their signals (A3: at approximately at $7 \mathrm{~s}$; A4: at approximately $22 \mathrm{~s}$ ). These times corresponded to the times when the bolts were released at the two supports. The perturbations observed in the recorded signals indicated that the damage was caused by a sudden reduction in the support rigidity.

(a)

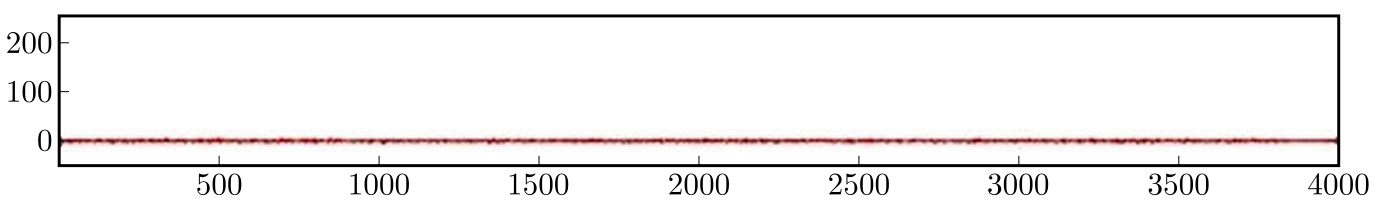

(b)

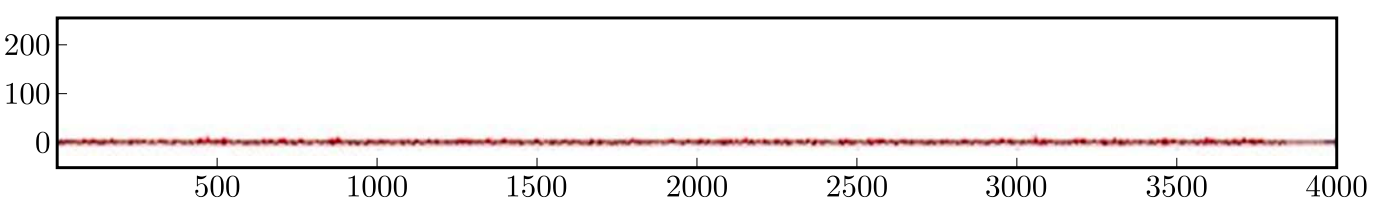

(c)

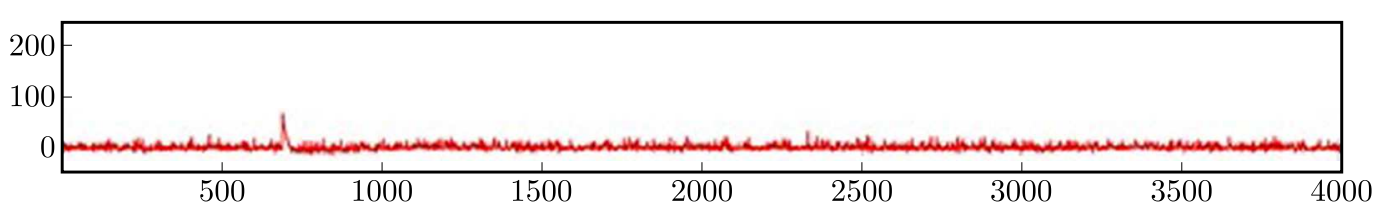

(d)

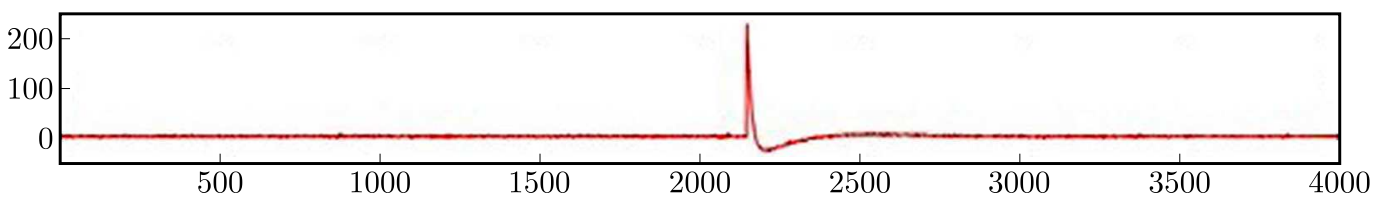

Fig. 5. Response accelerations when damage occurred at S3 and S4 for $l / 22.4$ (multiple damage): (a) sensor A1, (b) sensor A2, (c) sensor A3, (d) sensor A4 
In the cases considered above, the perturbations in the accelerations recorded by sensors A2 (for single damage), and A3 and A4 (multiple damage) corresponded to the times at which the bolts were removed from supports S2, S3, and S4, respectively. The other sensors in each case did not show obvious perturbations in their recorded accelerations. However, in practice, an unavoidable noise may lead to false damage detection. This situation becomes more obvious in practice, especially during field tests. This was experienced in many studies involving bridges (Siringoringo and Fujino, 2008) and buildings (Bakir et al., 2012). Some previous studies had to implement a non-probabilistic approach to dealing with noisy signals (Padil et al., 2017; Abdulkareem et al., 2018). A major advantage of WT methods over other conventional methods is its ability of denoising signals. This can be achieved by using a soft threshold shrinkage procedure (Ziopaja et al., 2011). The response measurements presented in this Section show that the distance between the point of damage (to a support) and sensor location plays an important role in capturing damage prints. The nearer the sensor to the damage location, the higher the chances of capturing the damage prints.

\subsection{Damage detection using continuous wavelet transform}

The response accelerations recorded at all supports for the two damage cases were decomposed by using CWT. The damage location was indicated by a sharp transition in the transformed response spatial variation using Eq. (2.5). CWT was applied by selecting the Gaussian wavelet as the mother wavelet. This was based by the trial and error method (Reda Taha et al., 2006). The selected WT modulus scale was $s=1-64$. Figures 6 to 8 show the results of the WT moduli of the three distance ratios of the single damage case, whereas Fig. 9 shows the multiple damage case with the best distance ratio. The $x$-axis represents the position in relation to time, while the $y$-axis represents the scale. The coefficient lines that show the changes in the magnitude of the obtained wavelet coefficient are also included. The scale of the coefficient lines $a$ is 32 .
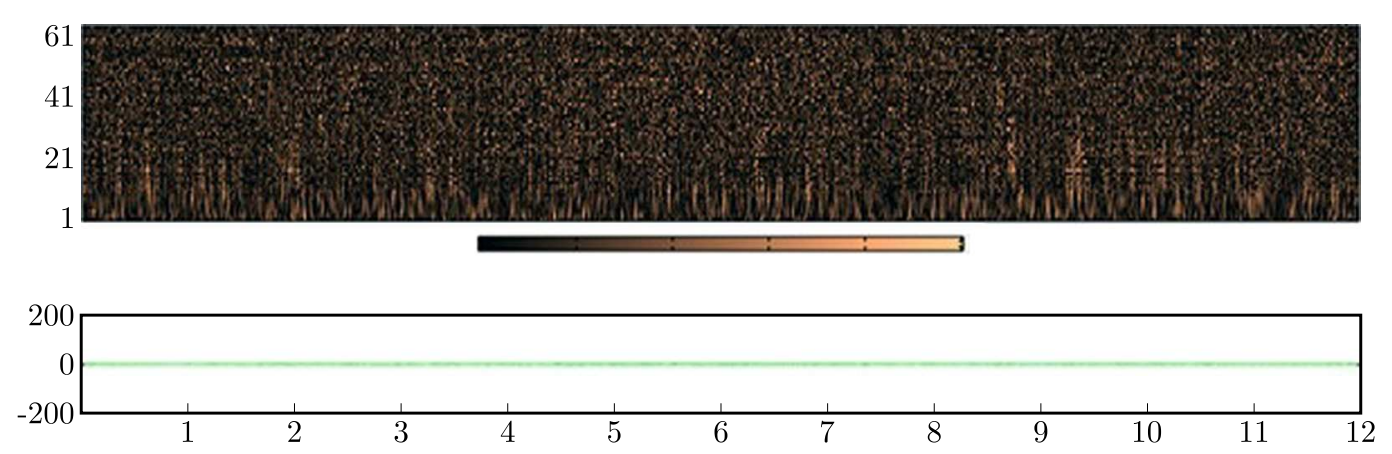

Fig. 6. WT modulus of response accelerations when damage occurred at $\mathrm{S} 2$ for $l / 2$ (single damage)

Figures 6 and 7 show the WT moduli of sensors installed when a single damage was imposed (S2) and the distance ratios were $l / 2$ and $l / 4$, respectively. In Fig. 6 , the decomposed signal measured at $280 \mathrm{~mm}$ from each support shows no spike in the WT modulus to indicate the damage. Figure 7 shows the WT modulus of the response accelerations measured at the distance ratio $l / 4$ when one sensor was placed $140 \mathrm{~mm}$ from each support. The damage was single and occurred at suppport S2 at $58 \mathrm{~s}$. The four WT moduli of the four measured accelerations show no damage spike. The minor peaks shown in the acceleration response signal failed to indicate any damage spike in the WT moduli. Similarly, the increase in acceleration, recorded by sensor A2, also did not indicate any damage spike.

In Figs. 8 and 9, the measured distance ratio was $l / 22.4(25 \mathrm{~mm})$. Clear spikes could be observed in the WT moduli of the sensor close to the damage supports. For example, in Fig. 8, for a single damage case at S2, only the WT modulus of sensor A2 (Fig. 8b) shows a spike. This 
(a)
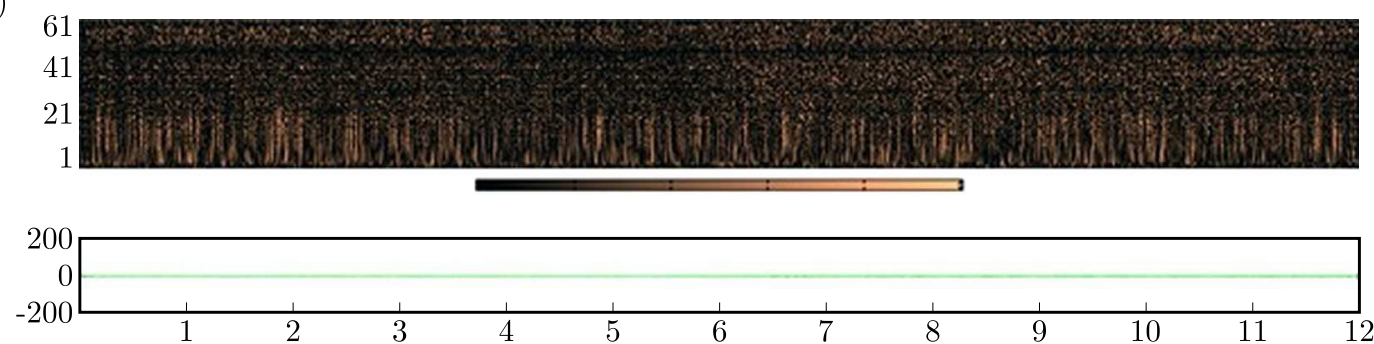

(b)
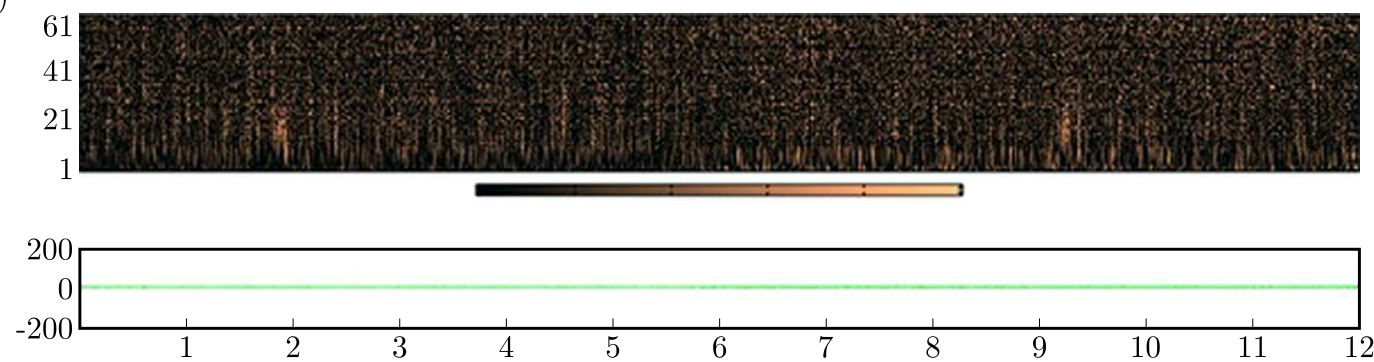

(c)
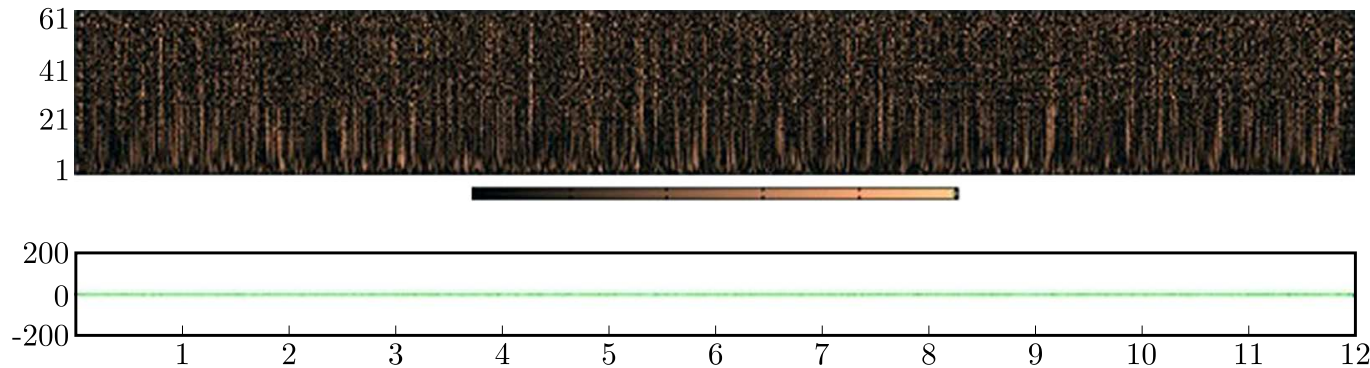

(d)
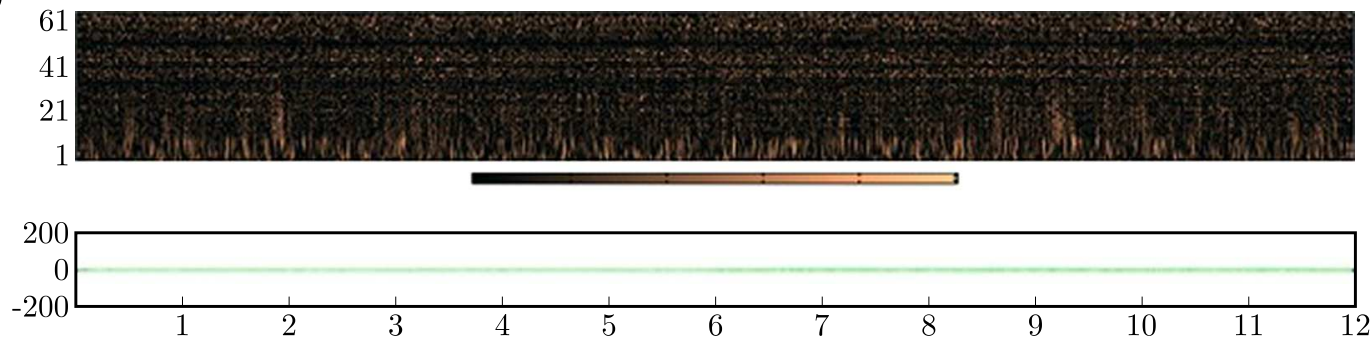

Fig. 7. WT modulus of response accelerations when damage at $\mathrm{S} 2$ for $l / 4$ (single damage):

(a) sensor A1, (b) sensor A2, (c) sensor A3, (d) sensor A4

spike shows damage occurrence in S2 at approximately $37 \mathrm{~s}$. The other WT moduli show no clear perturbations in the transformed signals, indicating that no damage occurred at supports S1, S3 and S4. This indicates that the method is able to detect the presence of the single damage, which occurred at S2. Figures 9 shows the results of the WT modulus of the multiple damage case. The decomposed signals show that the spikes only appear in the signals recorded by sensors A3 and A4 (Figs. 9c and 9d), showing that the method is able to detect the damage at supports S3 and S4, which are actual damage locations. The results presented above verified the efficiency of CWT in detecting singularities in signals. The damaged support is easily identified, and in practice, corrective measures can be taken immediately to prevent a structure from collapsing. This approach also prevents the acquisition of false data for SHM, as the support conditions alter vibration responses of the structures. The results of the proposed technique can facilitate the scrutiny of possible causes of damage and adoption of required preventive measures, because not only the damage locations but also the times at which damage occurred were identified. 
(a)
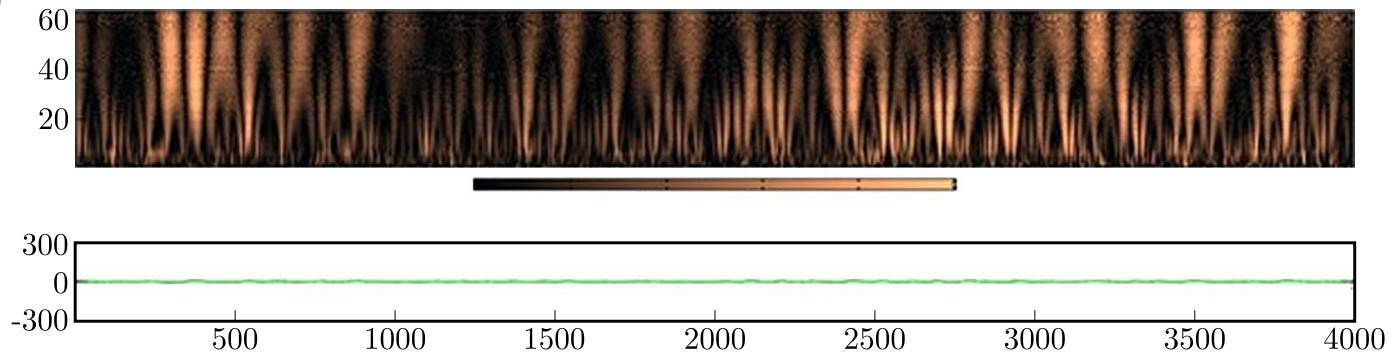

(b)

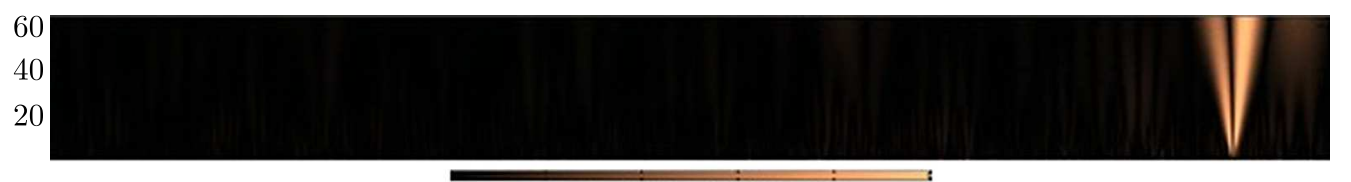

(c)
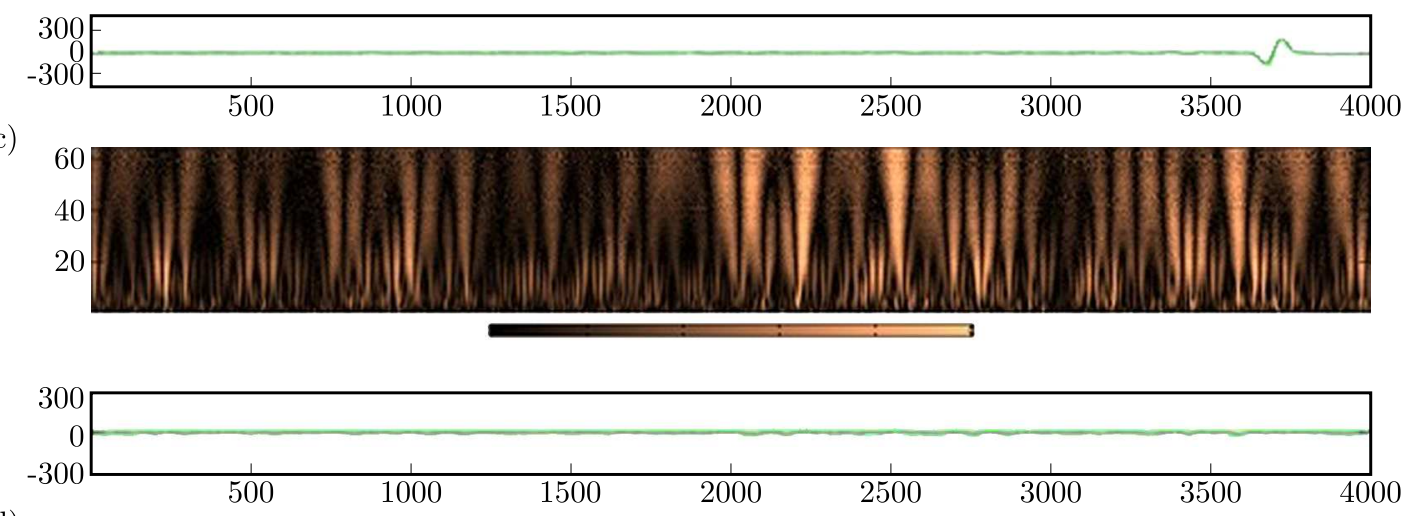

(d)
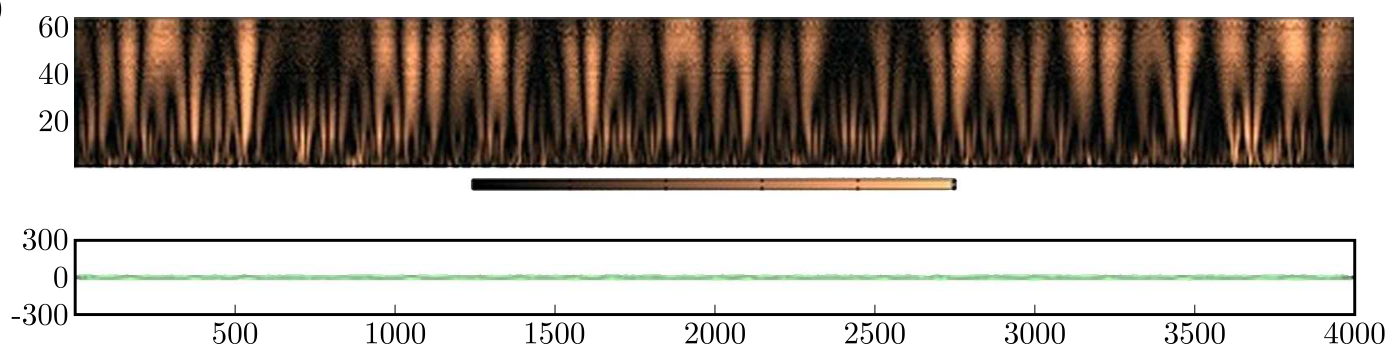

Fig. 8. WT modulus of response accelerations when damage occurred at S2 for 1/22.4 (single damage): (a) sensor A1, (b) sensor A2, (c) sensor A3, (d) sensor A4

\subsection{Effect of scale on damage detection}

The performance of WTs depends on the scale selected for decomposition of the signal. Gentile and Messina (2003) stated that an appropriate wavelet and scale selection is an efficient approach when data is noisy. They concluded that the finest scale is not the best choice when the data is noisy. Similarly, Rucka and Wilde (2006) reported that scale values required for experimental mode shapes were larger than those required for numerical, and that large scales could only detect large variation in the signal. However, Gaussian wavelet shows clear modulus maxima when the scale decreases (Mallat, 1998). Here, the effect of the scale was examined to investigate the sensitivity of damage signature to larger scales. The signals in the damage scenarios are decomposed using CWT at larger scales using the same Gaussian wavelet. The sampling interval time was $0.01 \mathrm{~s}$. CWT was performed using scale ranges $s=64-128$ and 200300 . Only signals that were measured with the best distance ratios $(l / 22.4)$ and at damaged supports were considered. Figures 10-12 show the WT moduli of the signals at supports S2, S3, and S4, which were recorded by sensors A2, A3, and A4, respectively, when damage occurred in these supports. A comparison of these results (Figs. 10-12) with the corresponding results 
(a)
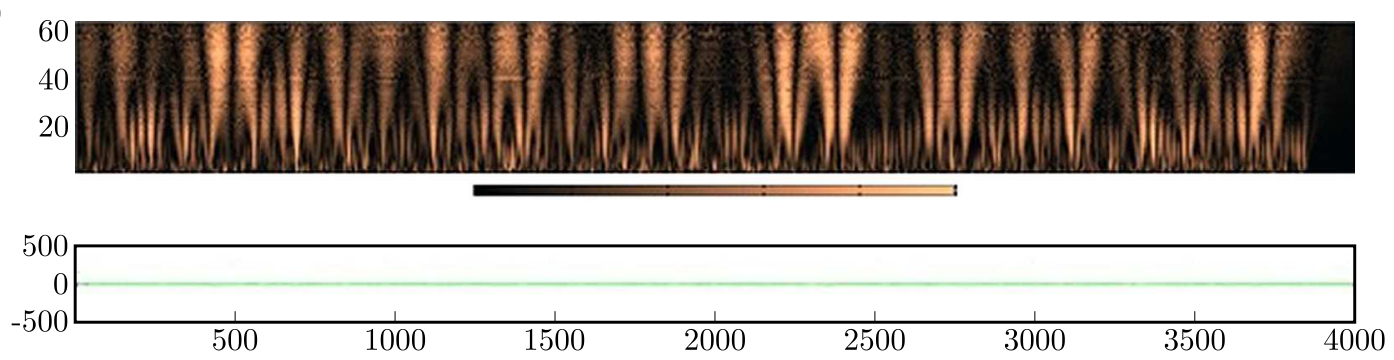

(b)
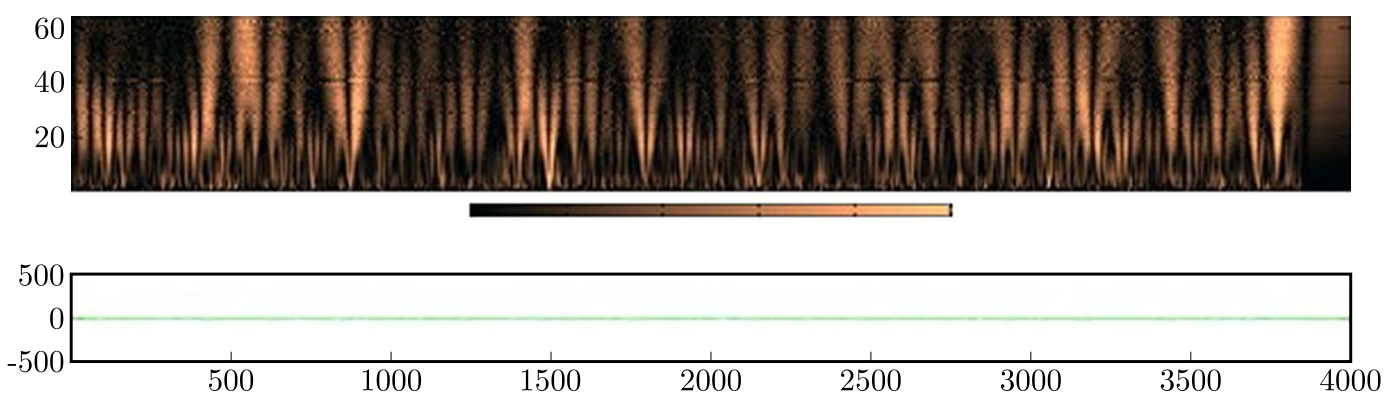

(c)
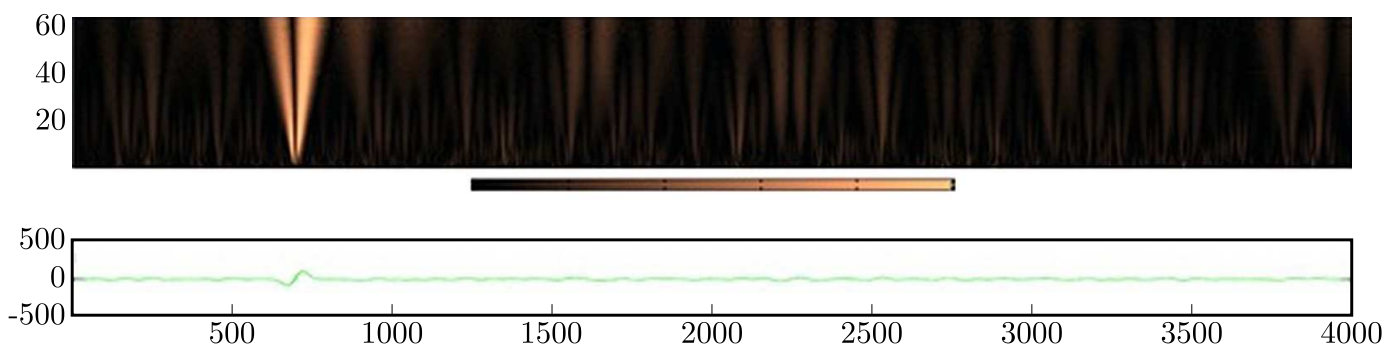

(d)
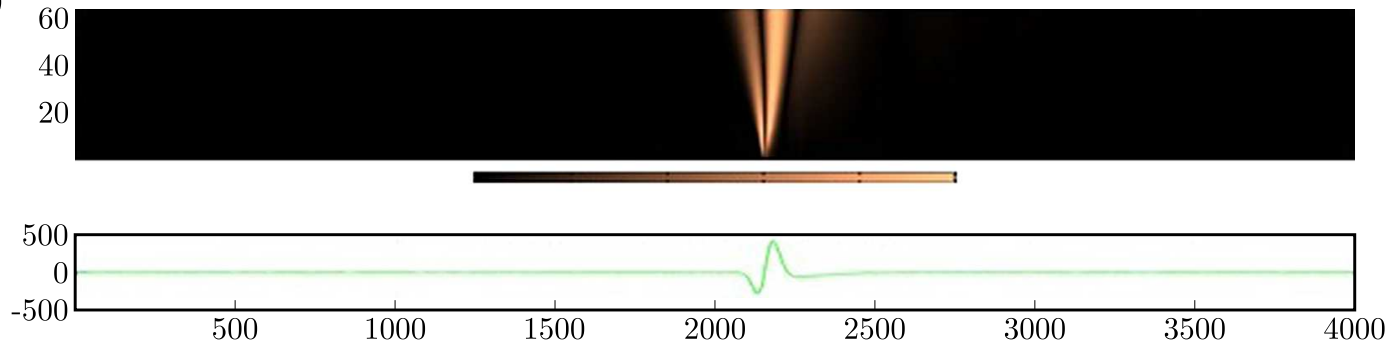

Fig. 9. WT modulus of response accelerations when damage at S3 and S4 for $l / 22.4$ (multiple damage):

(a) sensor A1, (b) sensor A2, (c) sensor A3, (d) sensor A4

shown in Figs. 8 and 9 indicates that the application of larger scales reduces the sharpness of the spike, which may hinder the detection of clear spikes in the WT modulus. For example, consider the damage at suppport S3 in the case of multiple damages. Figure 9c with a scale of 1-64 clearly indicates the position of the spike; the clarity of the spike decreases as the scale increases to 64-128 and 200-300, as shown in Fig. 11. The lowest scale provides the best clarity for spike identification. Thus, it can be concluded that CWT capability for damage detection decreases as the scale increases when the response accelerations are decomposed. Here, the best scale range was $s=1-64$.

\subsection{Discrete wavelet transform (DWT)}

This Section presents results of DWT analyses of the response acceleration signals, and covers the three distance ratios and both damage cases. The first to the fifth level detail coefficients of the decomposed signals for the two damage cases are shown in Figs. 13-16. Figure 13 shows the 

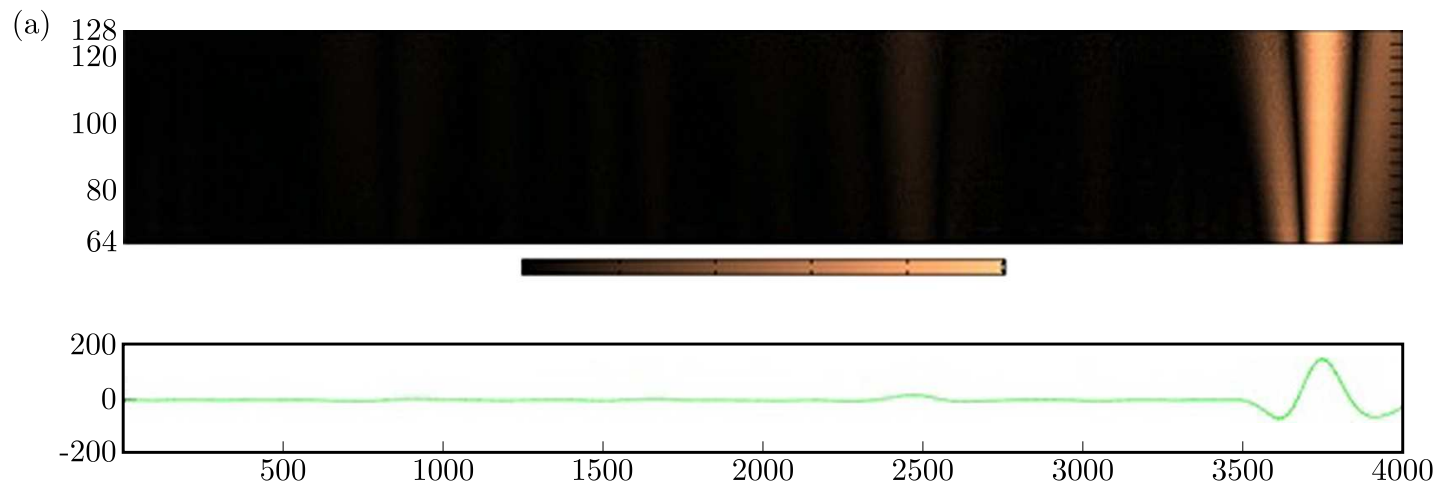

(b)
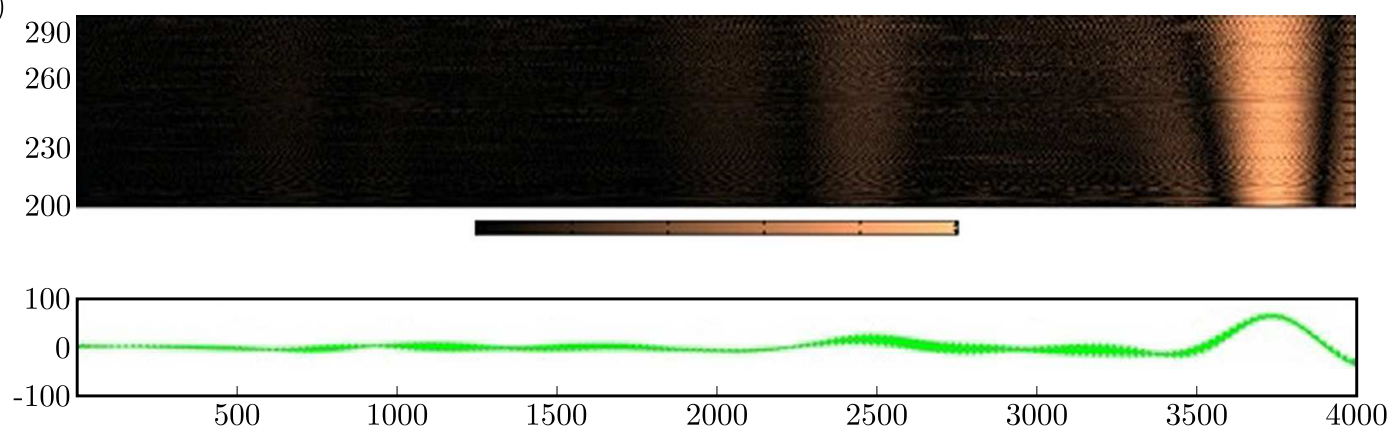

Fig. 10. WT modulus of sensor A2 for damage at S2: (a) scale $s=64-128$, (b) scale $s=200-300$

(a)
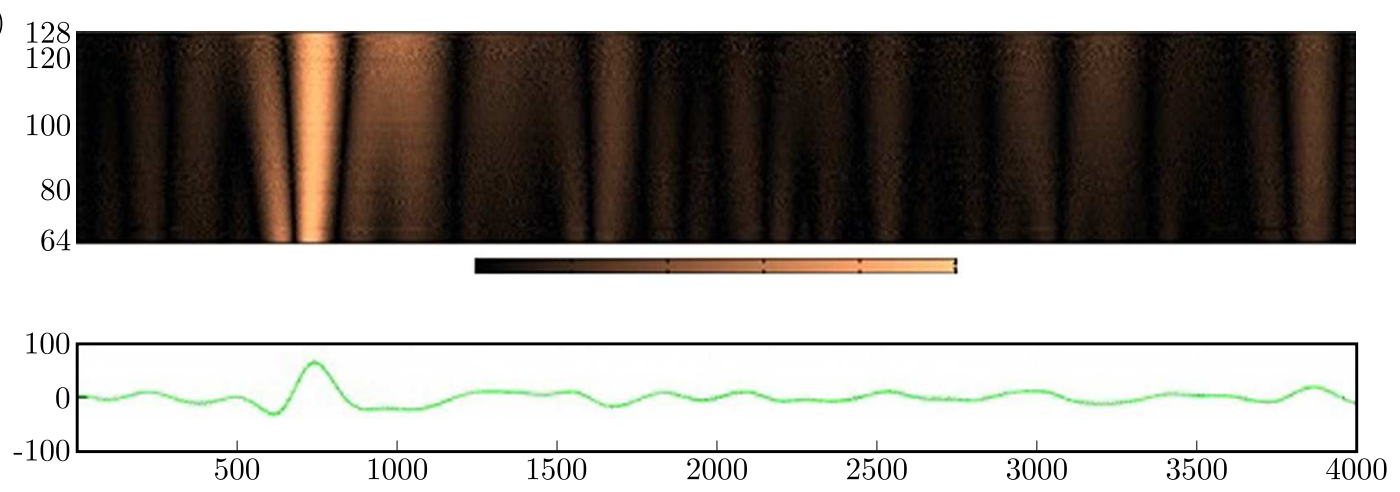

(b)

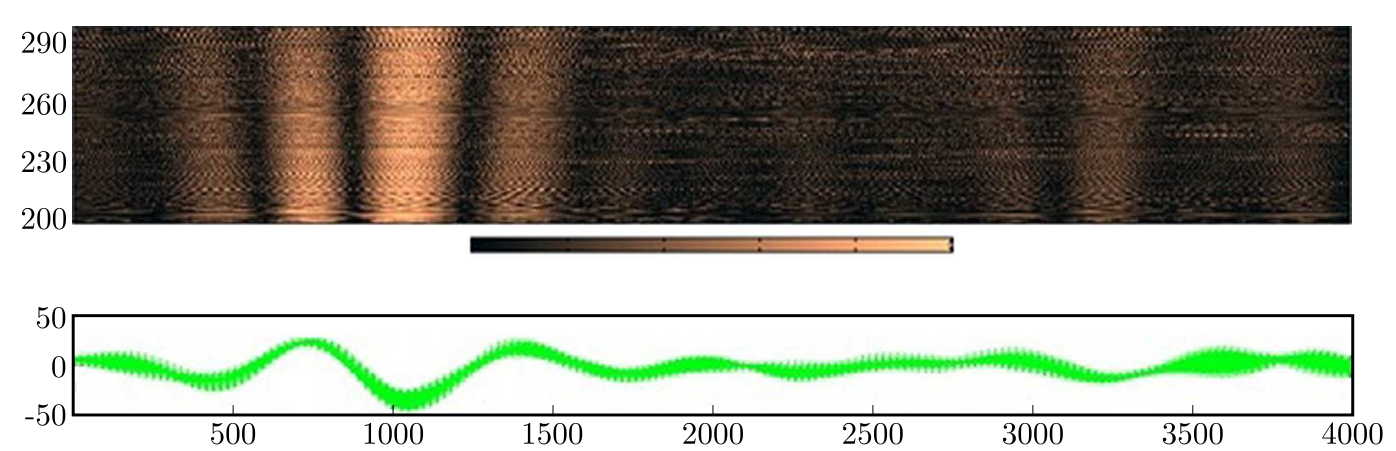

Fig. 11. WT modulus of sensor A3 for damage at S3: (a) scale $s=64-128$, (b) scale $s=200-300$

first to the fifth level detail coefficients of the decomposed signal when the response acceleration was measured at a distance ratio $l / 2$ (280 $\mathrm{mm}$ from each support). These coefficients show no clear spike to indicate the damage that occurred at approximately $58 \mathrm{~s}$ at suppport S2. This signal does not possess the damage print needed to indicate the damage spike in the coefficient. 
(a)
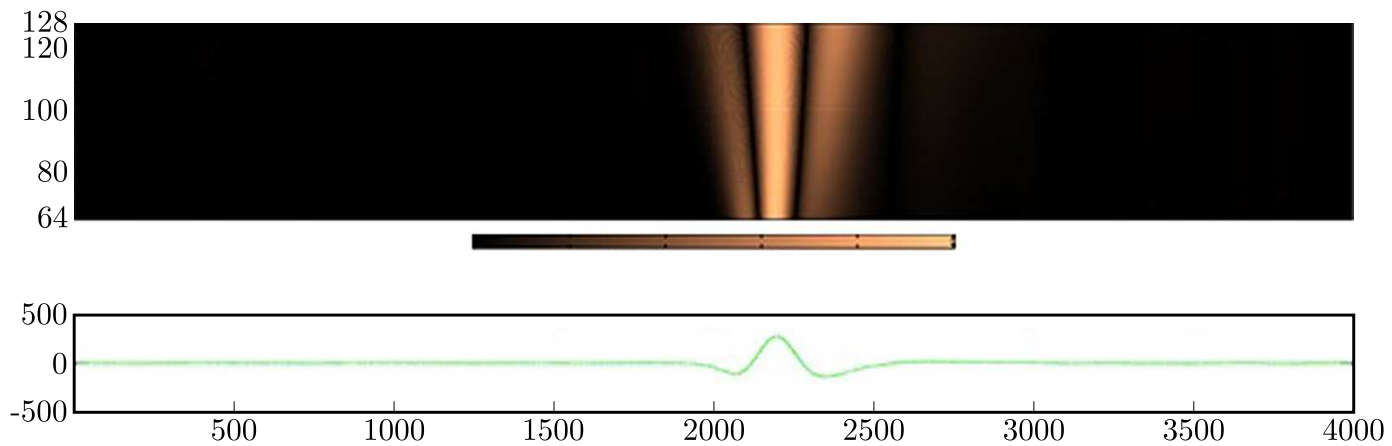

(b)
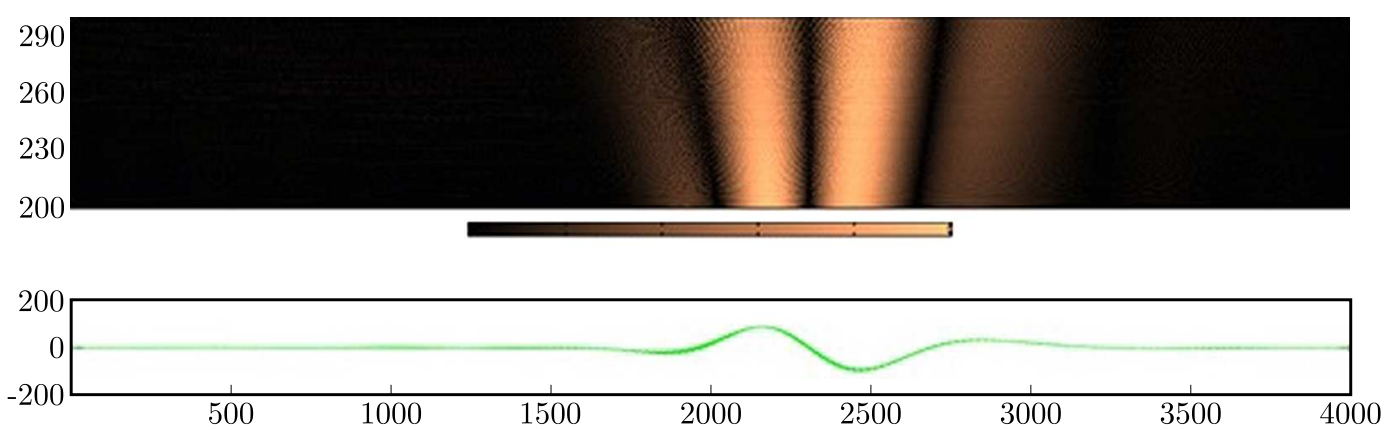

Fig. 12. WT modulus of sensor A4 for damage at S4: (a) scale $s=64-128$; (b) scale $s=200-300$

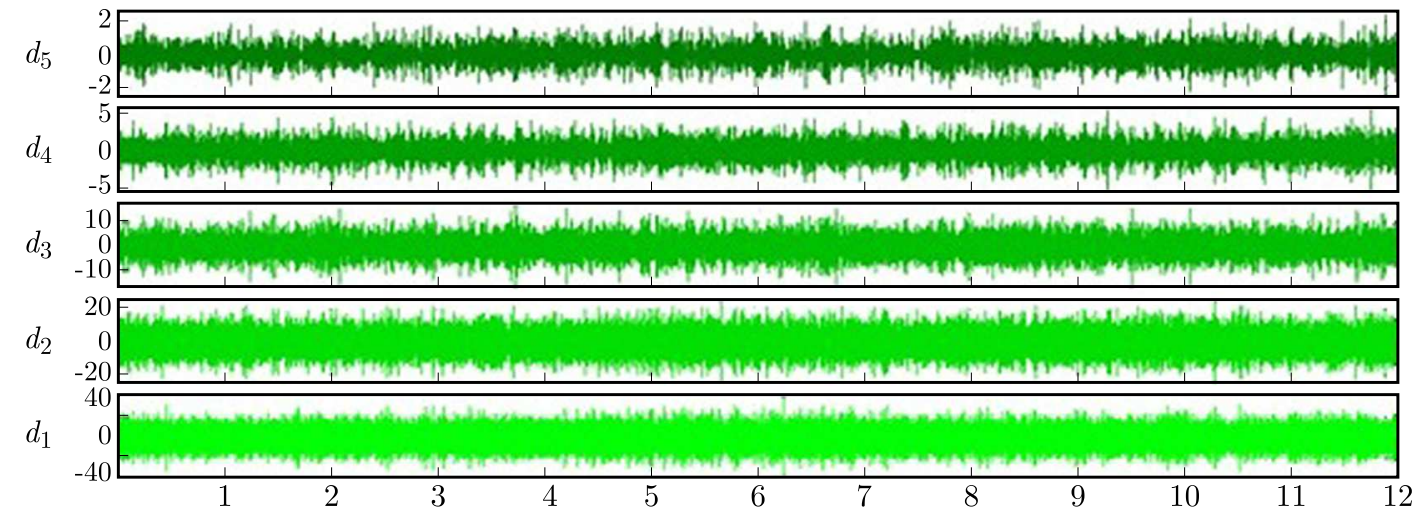

Fig. 13. First-to-fifth-level detail coefficients when damage occurred at S2 for $l / 2$ (single damage case)

Similarly, Fig. 14 shows the coefficients of response accelerations measured using four sensors at the distance ratio $l / 4$ (140 $\mathrm{mm}$ from each support). The results indicate that the damage was also undetected by the four sensors when it occurred at suppport S2. The absence of the spike in coefficients shows that the minor peaks in the response acceleration measured by the four sensors indicate the presence of noise. The non-detection of damage at $l / 2$ and $l / 4$ indicates that the distance between the damage and sensor location plays a vital role in damage detection at supports when DWT is applied.

From Figs. 15 and 16, the decomposed signals of the two damage cases at locations of damage have spikes at the time of damage occurrence. The indication of damage occurrence, location and time by DWT-analyzed signal coefficients correspond to the results obtained from the CWT coefficients. Figure 15 shows the first-to-fifth-level detail coefficients of the decomposed response accelerations measured when damage (single) occurred at suppport S2. Clear spikes are observed only in Fig. 16b, indicating damage occurrence at suppport S2. Figure 16 shows the first-to-fifth-level detail coefficients of the decomposed response accelerations measured when damage (multiple) occurred at supports S3 and S4. Figures 16c and 16d show clear spikes in the detail 
(a)

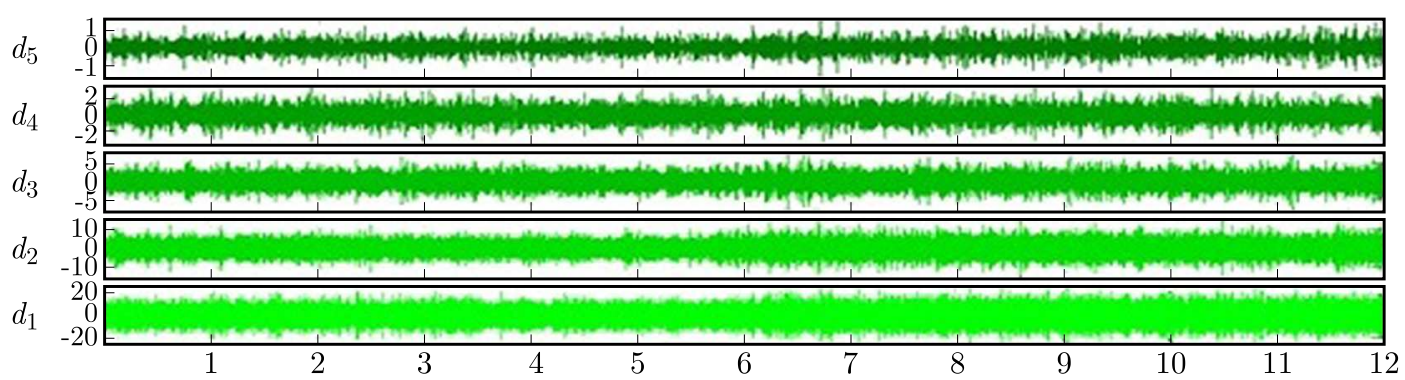

(b)

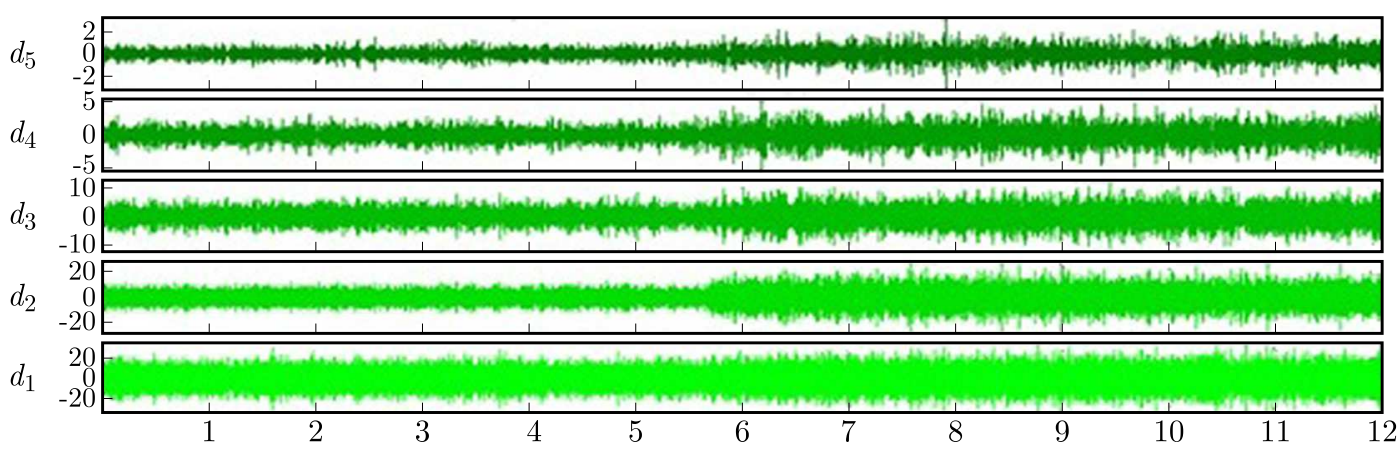

(c)

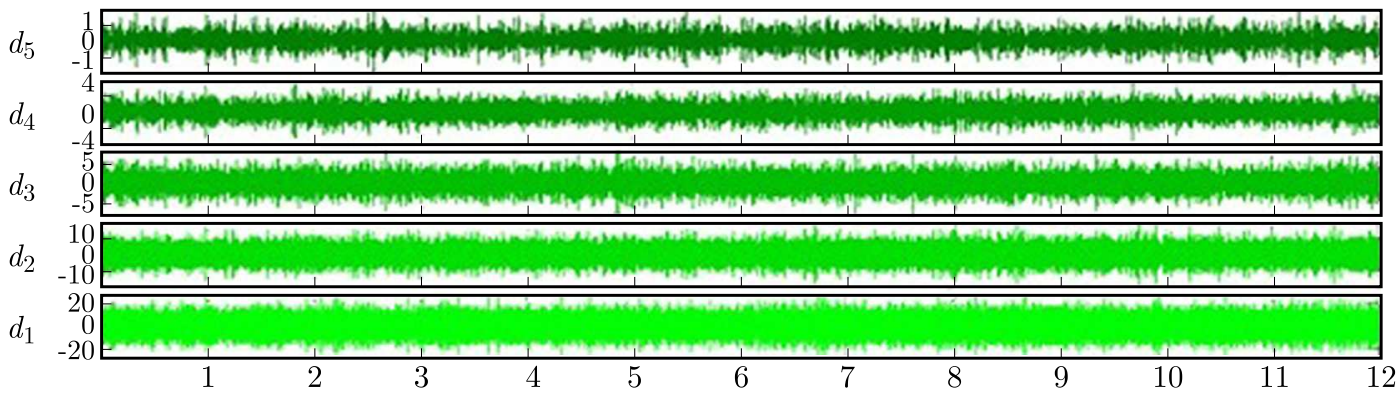

(d)

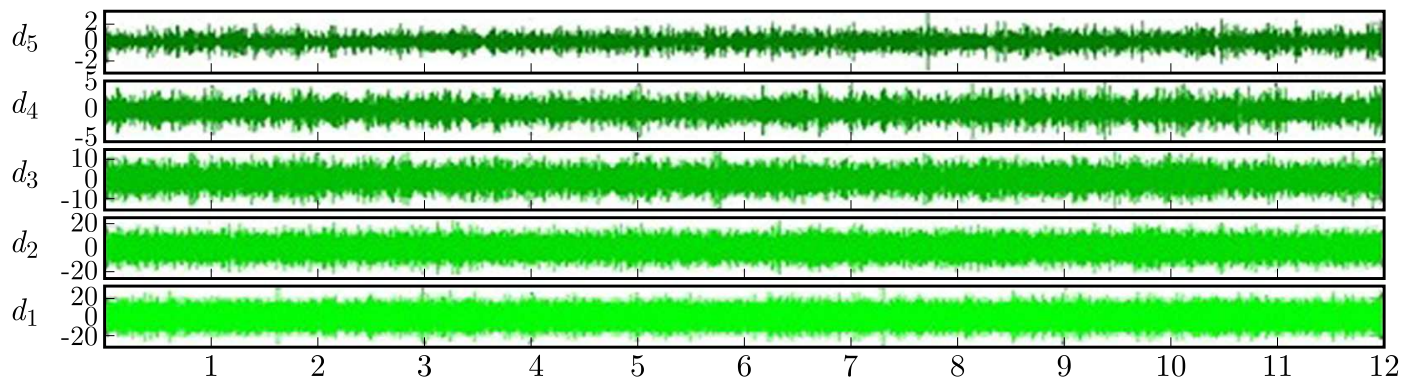

Fig. 14. First-to-fifth-level detail coefficients when damage occurred at S2 for $l / 4$ (single damage case): (a) sensor A1, (b) sensor A2, (c) sensor A3, (d) sensor A4

coefficients, which indicate damage occurrence at supports S3 and S4, respectively. Further, it can be observed in Fig. 15b that while the second, third, and fifth level detail coefficients have spikes indicating damage, the first level detail coefficient has no spike, whereas the spike for the fourth level is not clear. In Fig. 16c, the second, third and fourth level detail coefficients indicate damage, whereas the first and fifth level detail coefficients show no clear spike. Figure 16d clearly shows spikes for all five levels of detail coefficients.

The results presented in this Section confirm the capability of DWT for damage detection. In general, the two damage cases (single and multiple) are accurately detected, although the detail coefficients at some levels show no spikes to indicate damage. Therefore, it is advisable to analyze the signals using several levels of the detail coefficients. The results obtained by applying CWT in this test seem to be better compared to those obtained from DWT. While damage locations and times are defined and detected in CWT decompositions, DWT provides inaccurate results 
(a)

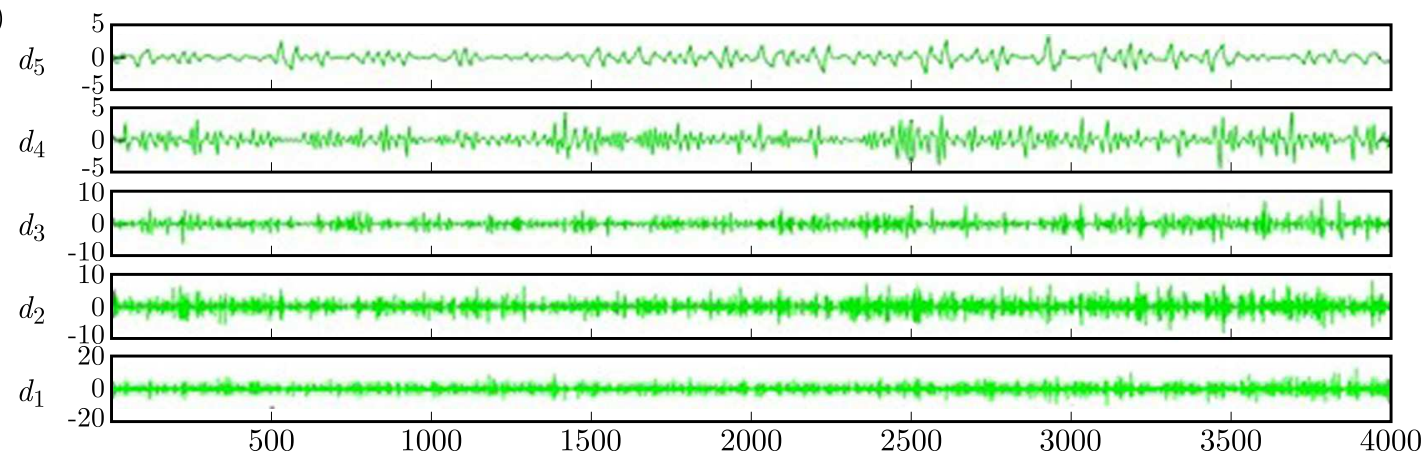

(b) $d_{5} \quad 100+00$

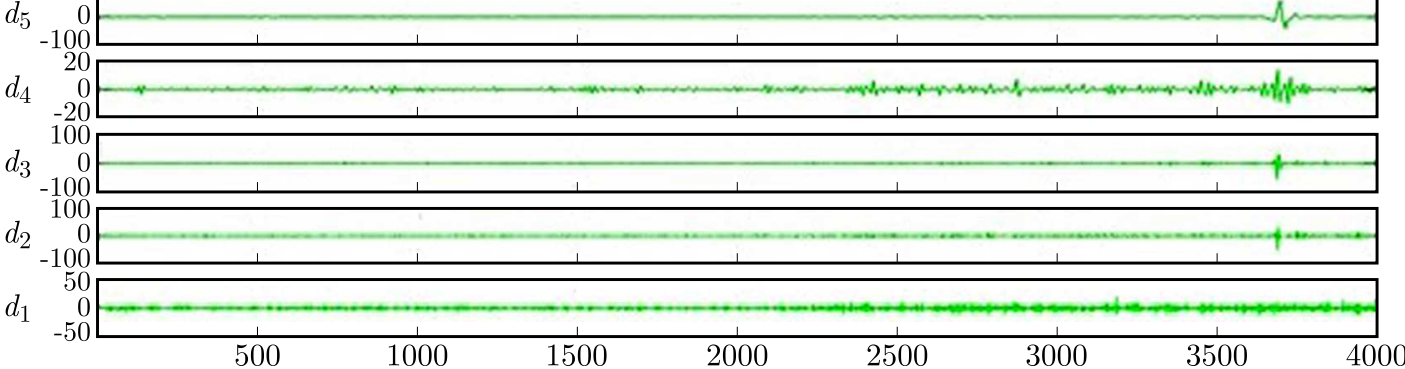

(c) 5 , 5

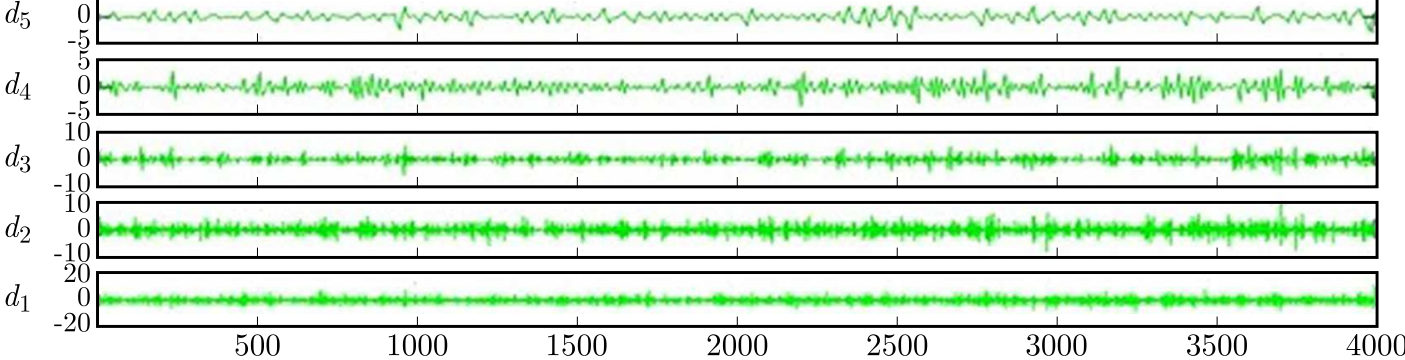

(d) $d_{5} \quad 05$ -

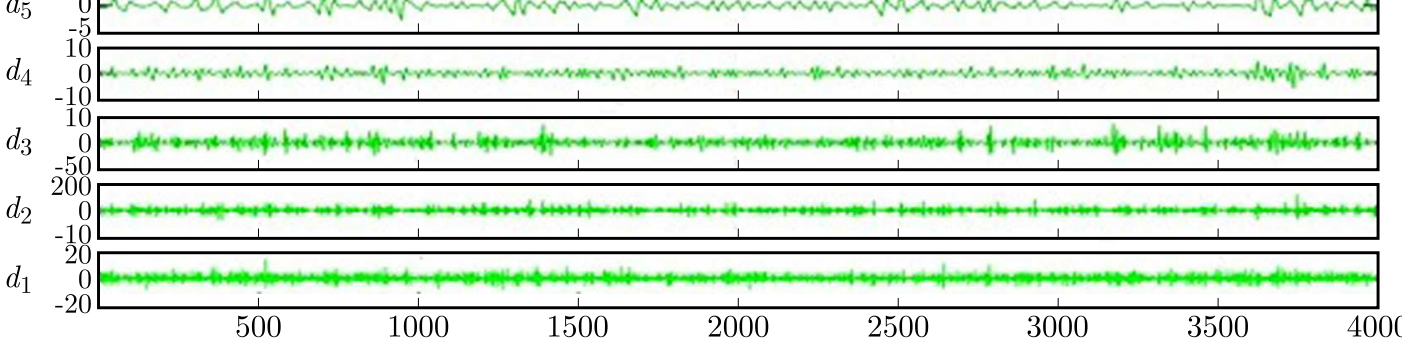

Fig. 15. First to fifth level detail coefficients when damage occurred at S2 (single damage case): (a) sensor A1, (b) sensor A2, (c) sensor A3, (d) sensor A4

in some instances. The first level detail coefficient failed to detect damage at suppport S2 when the signal recorded by A2 was decomposed (Fig. 15b), and this is similarly seen in the multiple damage case at suppport S3 (Fig. 16c).

\section{Conclusions}

This paper presents the application of response acceleration for assessing the support condition of a structure. Single and multiple damage cases were considered. Damage was induced by releasing bolts at the supports. It was observed that damage at a support affects only the response acceleration measured at points close to the damaged support. The response accelerations at points close to the supports were measured and analyzed using CWT and DWT. The stiffness 
(a)

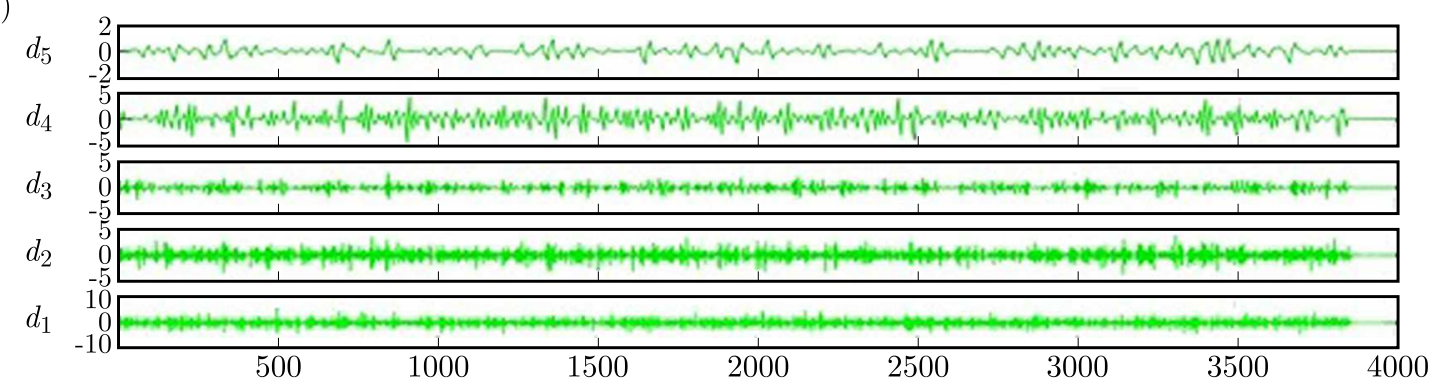

(b)

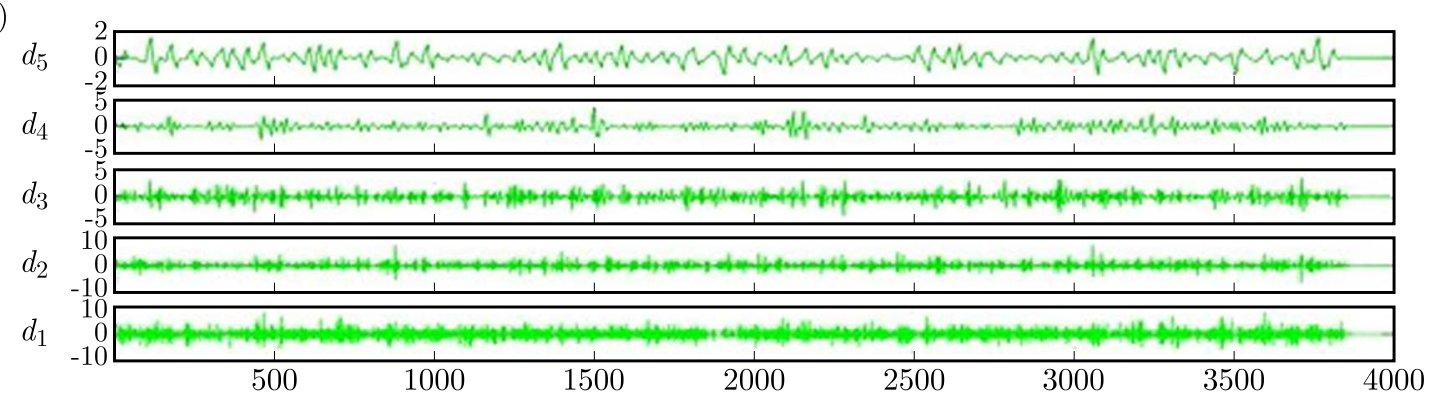

(c)

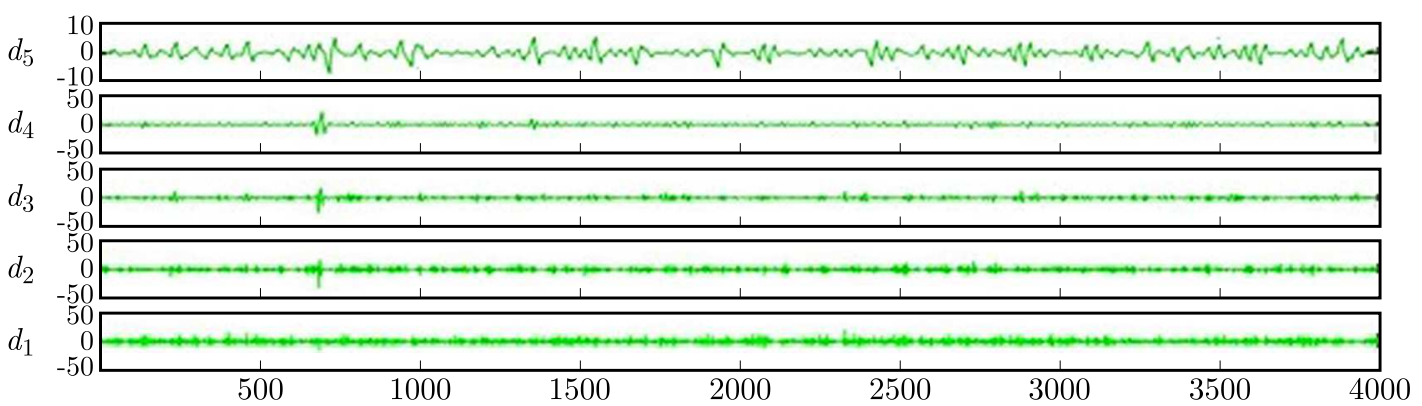

(d)

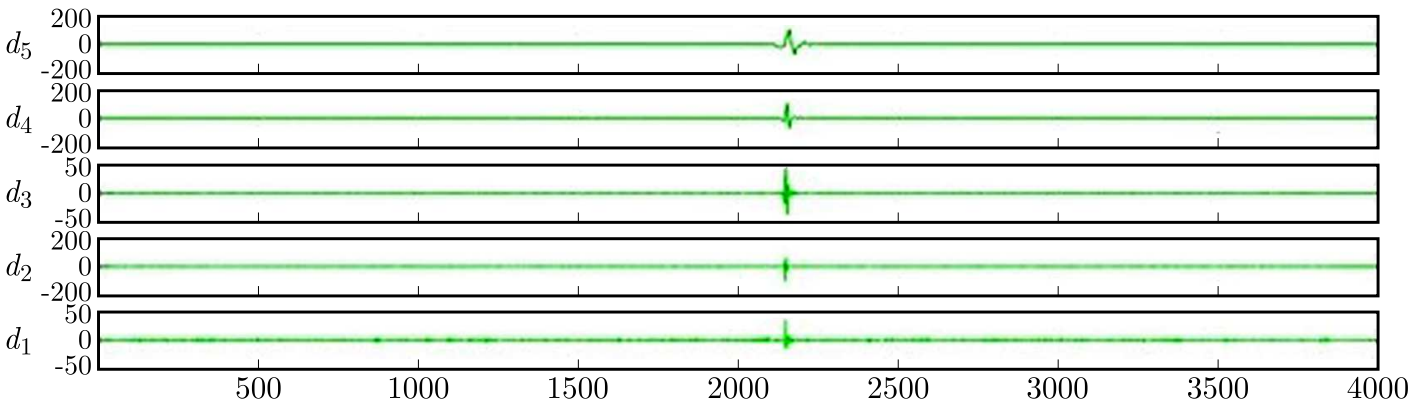

Fig. 16. First to fifth level detail coefficients when damage occurred at S3 and S4 (multiple damage case): (a) sensor A1, (b) sensor A2, (c) sensor A3, (d) sensor A4

loss due to bolt release at any damaged support had no effect on measurements at the undamaged supports. The effect of the damage decreased with the increasing distance from the damaged support. The effect of the stiffness of undamaged supports on the accelerations at nearby points exceeded the corresponding effect of the damaged supports. The damaged supports and time of damage were identified by decomposition of the response accelerations. The damage indicated by CWT analysis corresponded to that obtained from DWT analysis. The sensitivity of CWT to damage detection decreased as the scale increased when the acceleration was considered. DWT showed good damage identification capability, but the detail coefficients at some levels failed to indicate the damage. It should be noted that the effectiveness of this damage detection method was when the support damage occurred at the time the structural response signals were measured, stored and subjected to wavelet transformation. 


\section{Acknowledgements}

The authors thank the Ministry of Higher Education, Malaysia, and Universiti Teknologi Malaysia for their financial support through the FRGS (4F800) and HiCOE Grant (4J224).

\section{References}

1. Abdulkareem M., Bakhary N., Vafaei M., Noor N.M., Padil K.H., 2018, Non-probabilistic wavelet method to consider uncertainties in structural damage detection, Journal of Sound and Vibration, 433, 77-98

2. Bakhary N., Hao H., Deeks A.J., 2010, Substructuring technique for damage detection using statistical multi-stage artificial neural network, Advances in Structural Engineering, 13, 619-639

3. Bakir P., Eksioglu M., Alkan S., 2012, System identification of reinforced concrete building using the complex mode indicator function and the Hilbert transform technique, Testing And Evaluation, 40, 427-434

4. Cantero D., Basu B., 2015, Railway infrastructure damage detection using wavelet transformed acceleration response of traversing vehicle, Structural Control and Health Monitoring, 22, 62-70

5. Cao M., Xu W., Ostachowicz W., 2014, Damage identification for beams in noisy condition based on Teager energy operator-wavelet transform modal curvature, Journal of Sound and Vibration, 333, 1543-1553

6. Chen J., Rostami J., Peter T., Wan X., 2017, The design of a novel mother wavelet that is tailor-made for continuous wavelet transform in extracting defect-related features from reflected guided wave signals, Measurement, 110, 176-191

7. Douka E., Loutridis S., Trochidis A., 2003, Crack identification in beams using wavelet analysis, International Journal of Solids and Structures, 40, 3557-3569

8. Gentile A., Messina A., 2003, On the continuous wavelet transforms applied to discrete vibrational data for detecting cracks in damaged beams, International Journal of Solids and Structures, 40, 295-315

9. Hester D., GonzÁlez A., 2012, A wavelet-based damage detection algorithm based on bridge acceleration response to a vehicle, Mechanical Systems and Signal Processing, 28, 145-166

10. Hong J.C., Kim Y.Y., LeE H.C., LeE Y.W., 2002, Damage detection using the Lipschitz exponent estimated by the wavelet transform: applications to vibration modes of a beam, Solids and Structures, 39, 1803-1816

11. Lee Y.U., Kim Y.Y., LeE H.C., 2000, Damage detection in a beam via the wavelet transform of mode shapes, Transactions of the Korean Society of Mechanical Engineers A, 24, 916-925

12. Li J., HaO H., 2016, Health monitoring of joint conditions in steel truss bridges with relative displacement sensors, Measurement, 88, 360-371

13. Liu S., Du C., Mou J., Martua L., Zhang J., Lewis F.L., 2014, Diagnosis of structural cracks using wavelet transform and neural networks, NDT \& E International, 54, 9-18

14. Mallat S., 1998, A Wavelet Tour of Signal Processing, Academic Press, New York

15. Ovanesova A.V., SuÁrez L.E., 2004, Applications of wavelet transforms to damage detection in frame structures, Engineering Structures, 26, 39-49

16. PAdil K.H., BAKhary N., HaO H., 2017, The use of a non-probabilistic artificial neural network to consider uncertainties in vibration-based-damage detection, Mechanical Systems and Signal Processing, 83, 194-209

17. Reda Taha M.M., Noureldin A., Lucero J.L., Baca T.J., 2006, Wavelet transform for structural health monitoring: a compendium of uses and features, Structural Health Monitoring, 5, 267-295 
18. RuckA M., Wilde K., 2006, Application of continuous wavelet transform in vibration based damage detection method for beams and plates, Journal of Sound and Vibration, 297, 536-550

19. Siringoringo D.M., Fujino Y., 2008, System identification of suspension bridge from ambient vibration response, Engineering Structures, 30, 462-477

20. Staszewski W.J., 1998, Structural and mechanical damage detection using wavelets, The Shock and Vibration Digest, 30, 457-472

21. Vafaei M., Alih S.C., Rahman A.B.A., Adnan B.A., 2015, A wavelet-based technique for damage quantification via mode shape decomposition, Structure and Infrastructure Engineering, 11, 869-883

22. YAng J.N., Xia Y., Loh C.H., 2014, Damage identification of bolt connections in a steel frame, Journal of Structural Engineering, 140, 04013064

23. YAnG Y., NAGARAJAiAh S., 2014, Blind identification of damage in time-varying systems using independent component analysis with wavelet transform, Mechanical Systems and Signal Processing, 47, 3-20

24. Yu L., ZHU J., 2015, Structural damage detection of truss bridge under environmental variability, Applied Mathematics and Information Sciences, 9, 259-265

25. Yu Z., Xia H., Goicolea J.M., Xia C., 2016, Bridge damage identification from moving load induced detection based on wavelet transform and Lipschitz exponent, International Journal of Structural Stability and Dynamics, 16, 1550003

26. Zhang X., Feng N., Wang Y., Shen Y., 2015, Acoustic emission detection of rail defect based on wavelet transform and Shannon entropy, Journal of Sound and Vibration, 339, 419-432

27. Zhong S., Oyadius O., 2011, Crack detection in simply supported beams using stationary wavelet transform of modal data, Structural Control and Health Monitoring, 18, 169-190

28. Ziopaja K., Pozorski Z., Garstecki A., 2011, Damage detection using thermal experiments and wavelet transformation, Inverse Problems in Science and Engineering, 19, 127-153 